\title{
Rare left colic artery variation of the gut arterial system
}

\author{
S.A. Memar', A.M. Taylor², S. Ahuja², D.T. Daly²', Y. Tan² \\ ${ }^{1}$ A. T. Still University Kirksville College of Osteopathic Medicine, Kirksville, MO, United States \\ ${ }^{2}$ Centre for Anatomical Science and Education, Department of Surgery, Saint Louis University \\ School of Medicine, Saint Louis, MO, United States
}

[Received: 6 October 2021; Accepted: 15 November 2021; Early publication date: 26 November 2021]

\begin{abstract}
Most variations of the abdominal blood supply are related to branching of the coeliac trunk and superior mesenteric artery. This case details a remarkable variation in the branching pattern of the left colic artery (LCA) observed during routine cadaveric dissection of an 84-year-old male donor. An anomalous common trunk, originating from the common hepatic artery, gave rise to three branches: 1) an accessory posterior pancreaticoduodenal artery to the head of the pancreas and adjacent duodenum, 2) the dorsal pancreatic artery anastomosing with branches of the splenic artery, and 3) the LCA. The LCA descended between the splenic vein and superior mesenteric artery to supply the left colic flexure and form a collateral route with the middle colic artery by contributing to the marginal artery of Drummond. Knowledge of this variation is clinically relevant for surgical and radiological procedures in the abdomen. (Folia Morphol 2023; 82, 1: 198-204)
\end{abstract}

Key words: left colic artery, variation, inferior mesenteric artery, coeliac trunk, common hepatic artery, anomaly, anomalous, anastomosis, collateral, colon, liver, pancreas

\section{INTRODUCTION}

The coeliac trunk (CT), superior mesenteric artery (SMA), inferior mesenteric artery (IMA), and their collateral pathways are commonly subjected to anatomical variations of both origin and course due to their embryological development pattern [13-15, 18]. Of these three major aortic branches, the CT and SMA display higher rates of variation due to their development from the embryological celiac complex while the IMA is less prone to variation $[11,19]$. Variations of the left colic artery (LCA), which is normally the first branch from the IMA, are rare and the few reported instances often include an accessory LCA originating from the SMA or an absent LCA $[2,4,13,16]$. Knowledge of less commonly observed abdominal arterial variations, such as these, is clinically important for surgical and radiological interventions.

\section{Normal anatomy of the CT}

The $\mathrm{CT}$ is the first unpaired vessel arising from the ventral surface of abdominal aorta and it typically gives off three main branches: the left gastric artery (LGA), the splenic artery (SA), and the common hepatic artery (CHA).

The LGA travels cranially toward the oesophagus where it provides oesophageal branches before diving inferiorly along the lesser curvature of the stomach to anastomose with the right gastric artery. The SA immediately travels to the left after arising from the CT. The SA supplies blood to the pancreas as it travels

Address for correspondence: D.T. Daly, Assistant Professor, Centre for Anatomical Science and Education, Department of Surgery, Saint Louis University School of Medicine, Saint Louis, MO 63104, United States, tel.: 314-977-8027, fax: 314-977-5127, e-mail: daniel.daly@health.slu.edu This article is available in open access under Creative Common Attribution-Non-Commercial-No Derivatives 4.0 International (CC BY-NC-ND 4.0) license, allowing to download articles and share them with others as long as they credit the authors and the publisher, but without permission to change them in any way or use them commercially. 
along the organ's posterosuperior border. The SA then provides the short gastric arteries to the fundus of the stomach and the left gastroepiploic artery before reaching the spleen.

The third branch of the $\mathrm{CT}$ is the CHA which travels to the right to supply the liver, gallbladder, pancreas, and the proximal duodenum. Along its course, this artery changes its name to the proper hepatic artery after providing the gastroduodenal artery [15]. The right gastric artery may arise from either the common or proper hepatic artery to travel along the lesser curvature of the stomach and anastomose with the LGA. The proper hepatic artery then bifurcates into right and left hepatic arteries to supply the liver. This normal branching pattern of the CHA has been observed in $80 \%$ of the general population [14].

\section{Normal anatomy of the SMA}

The second major unpaired vessel originating from the ventral aspect of the abdominal aorta, approximately 1-2 cm below the origin of the CT, is the SMA. The inferior pancreaticoduodenal artery is typically the first branch off the SMA, followed by the middle colic, the right colic and the ileocolic arteries. From the left aspect of the SMA, numerous jejunal and ileal branches supply the small intestine [5].

\section{Normal anatomy of the IMA}

The IMA is the last major unpaired vessel of the abdominal aorta, originating from its left anterolateral aspect at the level of the third lumbar vertebra. The first branch of the IMA, the LCA, anastomoses with the middle colic and sigmoid arteries to provide circulation to the distal one-third of the transverse colon and the descending colon. Along its course, the IMA also gives off several sigmoidal arteries to supply the sigmoid colon before terminating as the superior rectal artery and supplying the superior rectum $[5,13,16]$.

\section{Purpose}

This report details a unique abdominal vascular variation of the hindgut in which the LCA originated from a common trunk arising from the $\mathrm{CHA}$, thereby creating an anastomotic connection between the $\mathrm{CT}$ and the IMA. Additional variations in the arterial supply to the duodenum and pancreas were observed in conjunction with this LCA variation. Knowledge of variations within the gut arterial system is relevant for diagnostic and interventional procedures of the abdominal viscera, especially the pancreas, duodenum, liver, and colon [14].

\section{CASE REPORT}

An 84-year-old male cadaver was received through the Saint Louis University Gift Body Programme of the Centre for Anatomical Science and Education (CASE) with signed, informed consent from the donor. The CASE Gift Body Programme abides by all the rules set forth by the Uniform Anatomical Gift Act.

\section{Common trunk from CHA}

During routine dissection, variations in the arterial supply to the pancreas, duodenum and hindgut-derivatives were observed. The CHA first provided an inferior branch which served as a common trunk for three arteries: an accessory posterior pancreaticoduodenal artery, the dorsal pancreatic artery, and the LCA (Fig. 1). This trifurcation occurred superior to the splenic vein, just to the left of the hepatic portal vein. Distal to the anomalous common trunk, the $\mathrm{CHA}$ displayed a typical branching pattern.

\section{First branch of the common trunk:}

\section{accessory posterior pancreaticoduodenal artery}

The first branch from the common trunk, described as an accessory posterior pancreaticoduodenal artery, travelled rightward along the posterosuperior border of the splenic vein before running deep to the left gastric vein and hepatic portal vein (Fig. 1). After traversing the width of the hepatic portal vein, the artery continued to the right along the posterior surface of the head of the pancreas before splitting into three branches to supply the duodenum and the head of the pancreas. The two superior branches anastomosed with the posterior superior pancreaticoduodenal artery, which originated from the gastroduodenal artery, as expected. The third branch was the largest and most inferior and anastomosed directly with the posterior inferior pancreaticoduodenal artery, which originated from the SMA, as expected. It was also noted the left gastric vein drained directly to the splenic vein instead of the hepatic portal vein as normally seen.

\section{Second branch of the common trunk: dorsal pancreatic artery}

The second branch, described as the dorsal pancreatic artery, coursed leftward, anterior to the splenic vein, before continuing as the inferior pancreatic 


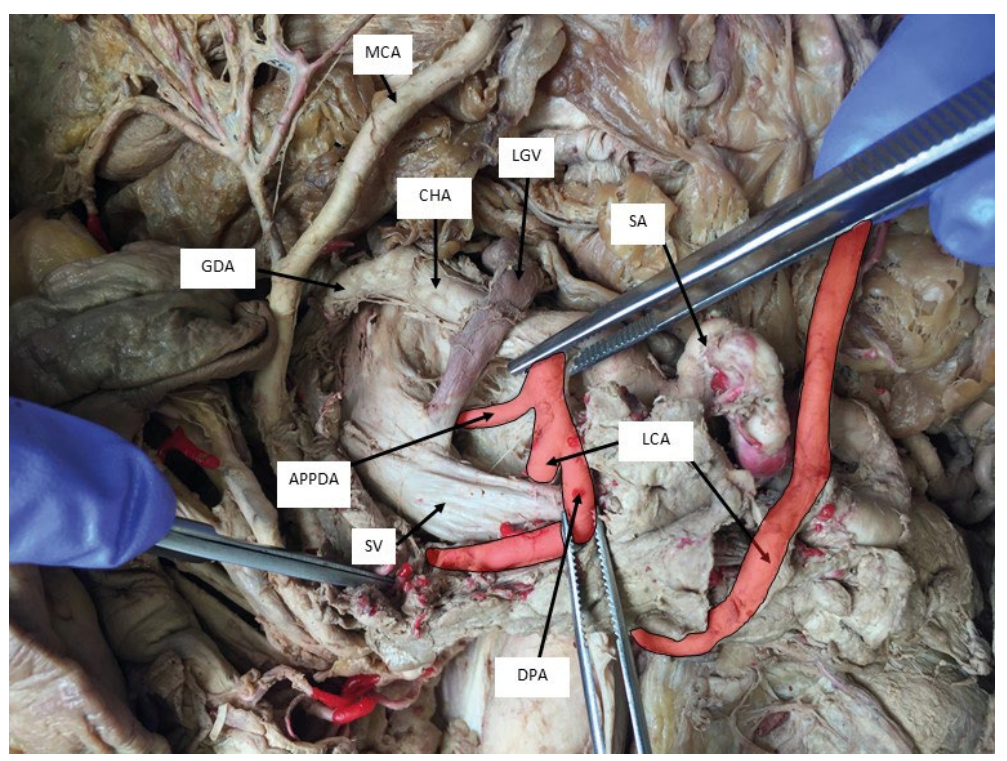

Figure 1. Anomalous common trunk branching from common hepatic artery. A common trunk (red) originated from the common hepatic artery and travelled inferiorly before trifurcating into an accessory posterior pancreaticoduodenal artery, the dorsal pancreatic artery, and the left colic artery. The trifurcation occurred superior to the splenic vein. The left colic artery travelled posterior to the splenic vein before continuing towards the splenic flexure to anastomose with the middle colic artery; APPDA — accessory posterior pancreaticoduodenal artery; CHA — common hepatic artery; DPA — dorsal pancreatic artery; GDA — gastroduodenal artery; LCA — left colic artery; LGV — left gastric vein; MCA — middle colic artery; SA — splenic artery; SV — splenic vein.

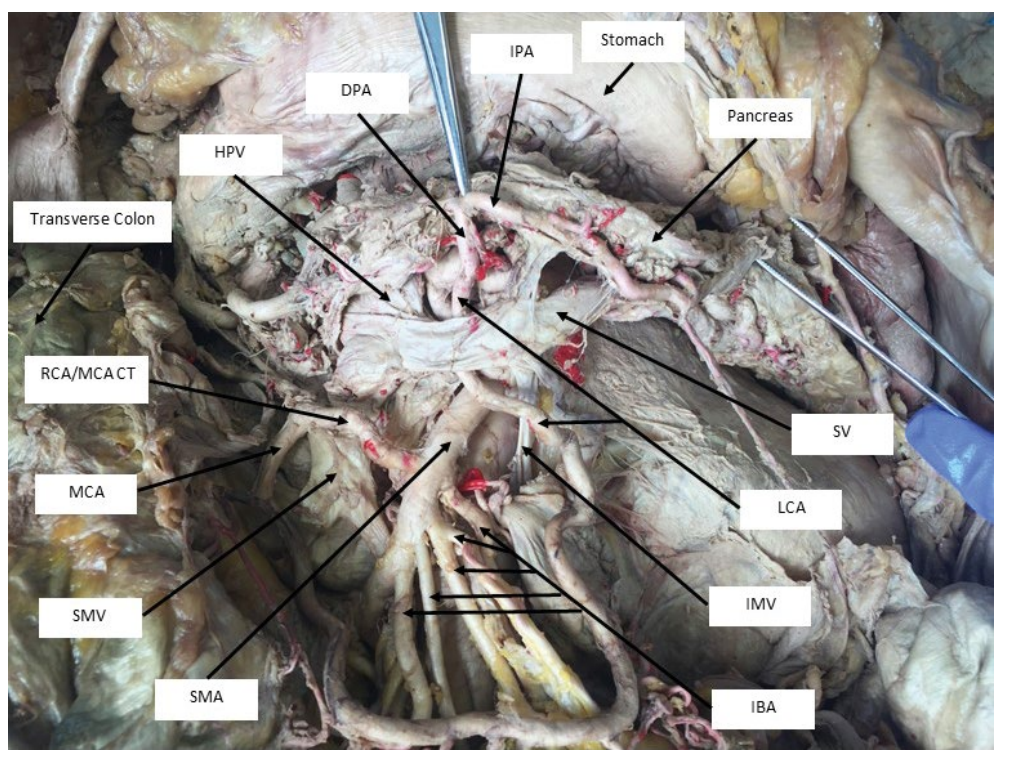

Figure 2. Courses of the dorsal pancreatic and left colic arteries. The dorsal pancreatic artery travelled leftward before continuing as the inferior pancreatic artery and anastomosing with branches of the splenic artery to supply the pancreas. The left colic artery travelled inferiorly between the splenic vein and superior mesenteric artery and was positioned to the right of the junction between the inferior mesenteric vein and the splenic vein before crossing over the inferior mesenteric vein. In this image, the transverse colon is pulled inferiorly and to the right, such that the middle colic artery is at the right of the image and the left colic artery is travelling inferiorly to anastomose with it; DPA — dorsal pancreatic artery; HPV — hepatic portal vein; IBA — intestinal branch arteries; IMV — inferior mesenteric vein; IPA — inferior pancreatic artery; LCA — left colic artery; MCA — middle colic artery; RCA/MCA CT — common trunk of the right and middle colic arteries; SMA — superior mesenteric artery; SMV — superior mesenteric vein; SV — splenic vein.

artery along the posteroinferior border of the pancreas (Fig. 2). While travelling toward the tail of the pancreas, it looped superiorly at two different points in the middle of the pancreatic body, thereby forming two anastomotic connections with branches of the SA within the pancreas. 


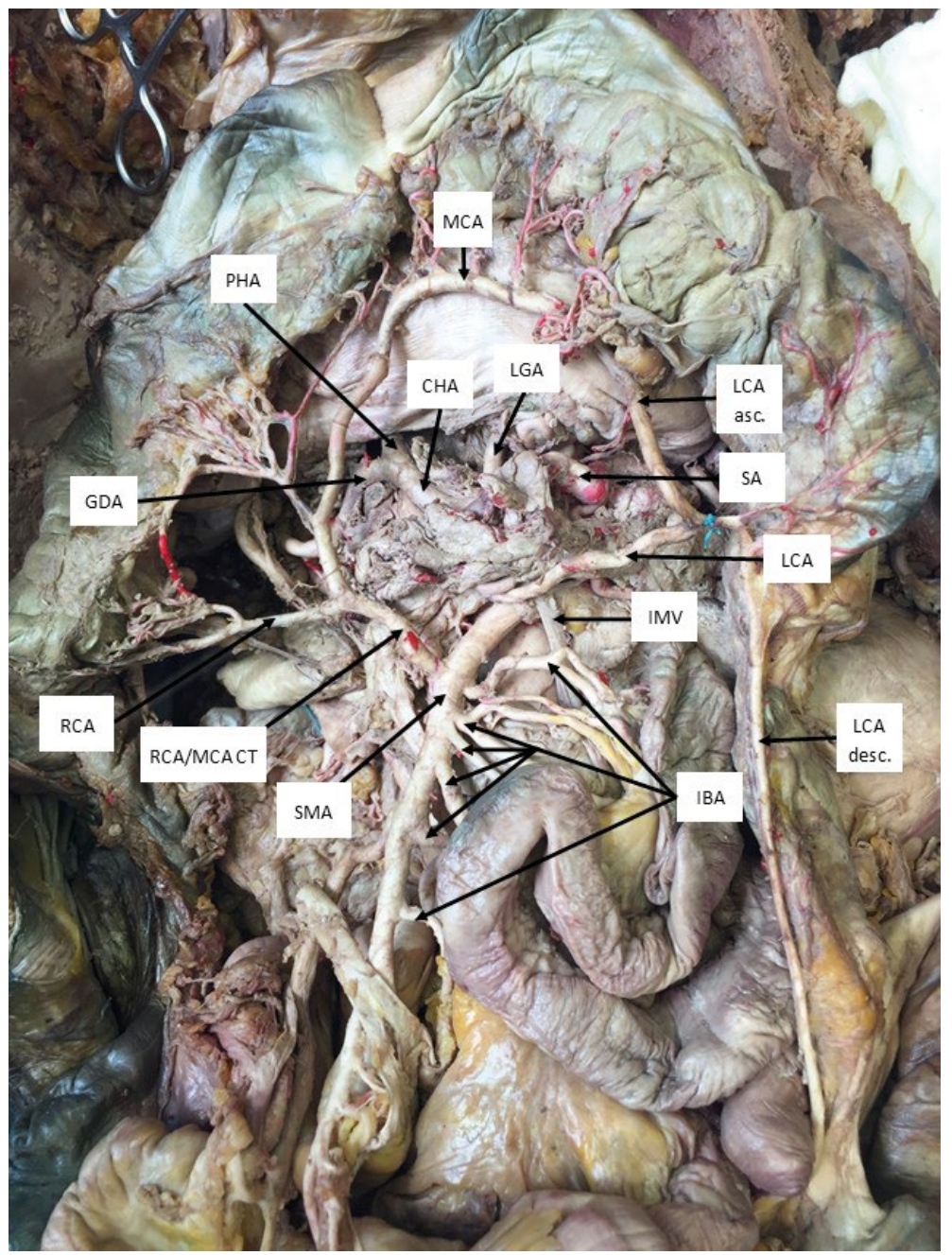

Figure 3. Left colic artery supplying hindgut with view of midgut. The left colic artery was positioned to the right of the junction between the inferior mesenteric vein and the splenic vein after coursing anterior to the superior mesenteric artery. It then passed anterior to the inferior mesenteric vein as it travelled leftward toward the splenic flexure, where it divided into ascending and descending branches. The ascending branch formed an anastomosis with the middle colic artery, which originated from a common trunk with the right colic artery. The descending branch continued inferiorly along the descending colon before anastomosing with the sigmoid branches of the inferior mesenteric artery (not pictured); CHA — common hepatic artery; GDA — gastroduodenal artery; IBA — intestinal branch arteries; IMV — inferior mesenteric vein; LGA — left gastric artery; LCA — left colic artery; LCA asc. — ascending branch of the left colic artery; LCA desc. — descending branch of the left colic artery; MCA — middle colic artery; PHA — proper hepatic artery; RCA — right colic artery; RCA/MCA CT — common trunk of the right and middle colic arteries; SA — splenic artery; SMA — superior mesenteric artery.

\section{Third branch of the common trunk:}

\section{left colic artery}

The most distal branch from the common trunk was the LCA, which travelled inferiorly, posterior to the splenic vein and anterior to the SMA (Figs. 1, 2). After passing over the inferior mesenteric vein, just below its junction with the splenic vein, the LCA coursed leftward towards the splenic flexure and divided into ascending and descending branches (Figs. 3, 4). The ascending branch, which had the larger diameter of the two branches, formed an anastomosis with the middle colic artery, which shared a common trunk with right colic artery from the SMA. The descending branch continued inferiorly along the descending colon before anastomosing with the sigmoid arteries. The sigmoid and superior rectal arteries originated from the IMA as expected. In this case, the marginal artery of Drummond was formed by the branches from the CT and the SMA and IMA instead of solely the SMA and IMA, as seen normally.

\section{DISCUSSION}

\section{Embryological causes and established categorisation of gut arterial variations}

The CT, SMA, and IMA are derived from a series of ventral vitelline arteries arising from the embryo- 


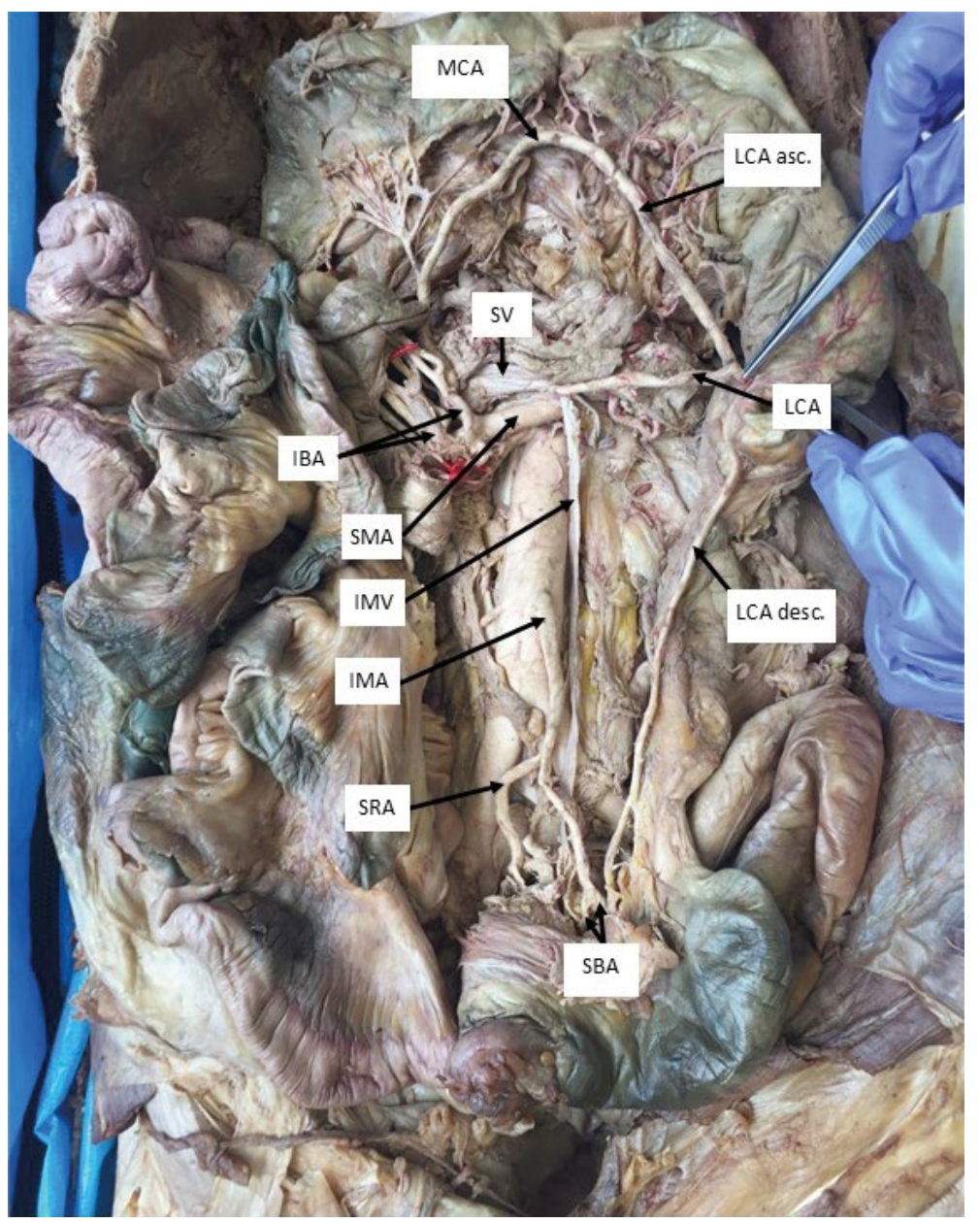

Figure 4. Left colic artery supplying hindgut. The ascending branch of the left colic artery anastomosed with the middle colic artery. The descending branch of the left colic artery anastomosed with the sigmoid branches of the inferior mesenteric artery. In this case, the marginal artery of Drummond was uniquely formed by the branches from the celiac trunk and the superior and inferior mesenteric arteries; IBA - intestinal branch arteries; IMA — inferior mesenteric artery; IMV — inferior mesenteric vein; LCA — left colic artery; LCA asc. — ascending branch of the left colic artery; LCA desc. — descending branch of the left colic artery; MCA — middle colic artery; SBA — sigmoid branch arteries; SMA — superior mesenteric artery; SRA — superior rectal artery; SV — splenic vein.

logical dorsal aortae to supply the fetal abdominal viscera [17]. The vitelline arteries are connected by an anastomotic channel that should regress to promote the development of the unpaired visceral arteries, though the persistence of vitelline remnants may explain variations in the vascular supply to this region $[8,17]$. Most abdominal vascular variations in the subdiaphragmatic aorta are related to the liver and kidneys through variations of the CT and SMA [17].

There are several classification systems used to describe intestinal arterial variations. Lipschutz, Adachi, and Morita independently classified the origins of the left gastric, splenic, common hepatic and superior mesenteric arteries $[1,6,10]$. Gamo et al. [6] observed cadaveric specimens and computed tomography images to categorize SMA branching patterns [6]. However, the current case cannot be analysed using the previous methods, as it either does not fit into the defined categories or it does not involve the mentioned branches.

Most relevant to the current case, Cirocchi et al. [4] conducted a systematic literature review of LCA anomaly case studies from 1953 until 2019 and categorised the LCA branching pattern from the IMA according to Latarjet's work: the LCA either originated independently or from a common trunk with sigmoidal branches $[4,20]$. However, in both cases, the LCA was observed to originate from the IMA, which differs from the current variation.

\section{Potential associated pathologies}

The anatomical variants observed in the current case could potentially be related to a number of pathological presentations. The LCA as observed in 
this case can be associated with compression of the splenic vein and resulting left-sided portal hypertension, which is a rare but life-threatening cause of upper gastrointestinal bleeding. Conversely, portal hypertension may compress the LCA in this unique case and block the blood supply to the distal colon. Additionally, the described vasculature could complicate procedures including pancreatectomy, pancreaticoduodenectomy, cholecystectomy, colectomy and hepatectomy [12]. Arteries supplying the abdominal viscera have also been investigated regarding their efficacy in bypass procedures, so an understanding of gut arterial anastomoses is necessary to determine if such a procedure is possible [3].

While the aforementioned complications are unique to this anatomical presentation, the common surgical concerns associated with any abdominal procedure remain. Examples include nephrectomy procedures which may be jeopardised by the LCA passing in close proximity to supply the left colic flexure and the potential for watershed ischemia at Griffith's point (which is the point of anastomosis between the middle and left colic arteries) [7, 9, 13]. In general, surgical procedures involving the aorta, liver, gallbladder, stomach, duodenum, pancreas, kidneys or lymph nodes may all be impacted by variations like the one presented in this case study.

\section{CONCLUSIONS}

This case study involves an anomalous common trunk branching from the $\mathrm{CHA}$ and providing the following three branches: an accessory posterior pancreaticoduodenal artery, the dorsal pancreatic artery, and the LCA. While arterial variations of the gut have been recorded in the past, such a connection between the CT and IMA has rarely been reported. Knowledge of atypical gut arterial supply, specifically to the duodenum, pancreas, and distal colon, is beneficial for clinicians to determine the feasibility of surgical procedures, thereby appropriately caring for patients.

\section{Acknowledgements}

We would like to thank the Gift Body Programme at the Centre for Anatomical Science and Education, Department of Surgery at Saint Louis University School of Medicine. This case report was presented in part at the 2021 Virtual AACA Annual Meeting of the American Association of Clinical Anatomists.

Conflict of interest: None declared

\section{REFERENCES}

1. Adachi B, Hasebe K, Daigaku K. Das Arteriensystem der Japaner. Kyoto, Kaiserlich-Japanische Universität zu Kyoto. Maruzen Co., Kyoto and Tokyo 1928.

2. Amonoo-Kuofi HS, el-Badawi MG, el-Naggar ME. Anomalous origins of colic arteries. Clin Anat. 1995; 8(4): 288-293, doi: 10.1002/ca.980080408, indexed in Pubmed: 7552967.

3. Calafiore A, Giammarco GDi, Teodori G, et al. Myocardial revascularization with multiple arterial grafts. Asian Cardiovasc Thoracic Ann. 2016; 3(3-4): 95-102, doi: 10.1177/021849239500300402.

4. Cirocchi R, Randolph J, Cheruiyot I, et al. Systematic review and meta-analysis of the anatomical variants of the left colic artery. Colorectal Dis. 2020; 22(7): 768-778, doi: 10.1111/codi.14891, indexed in Pubmed: 31655010.

5. De Martino RR. Normal and variant mesenteric anatomy. Springer, New York 2015.

6. Gamo $E$, Jiménez $C$, Pallares $E$, et al. The superior mesenteric artery and the variations of the colic patterns. A new anatomical and radiological classification of the colic arteries. Surg Radiol Anat. 2016; 38(5): 519-527, doi: 10.1007/ s00276-015-1608-3, indexed in Pubmed: 26728989.

7. Ke J, Cai J, Wen X, et al. Anatomic variations of inferior mesenteric artery and left colic artery evaluated by 3-dimensional CT angiography: Insights into rectal cancer surgery. A retrospective observational study. Int J Surg. 2017; 41: 106-111, doi: 10.1016/j.ijsu.2017.03.012, indexed in Pubmed: 28323157.

8. Kosaka M, Horiuchi K, Nishida K, et al. Hepatopancreatic arterial ring: bilateral symmetric typology in human celiaco-mesenteric arterial system. Acta Med Okayama. 2002; 56(5): 245-253.

9. Mann M, Kawzowicz M, Komosa A, et al. The marginal artery of Drummond revisited: a systematic review. Transl Res Anat. 2021; 24: 100118, doi: 10.1016/j. tria.2021.100118.

10. Morita M. Sekimen kyōfu no ryōhō. Jinbun, Kyōto 1935.

11. Murakami T, Mabuchi M, Giuvarasteanu I, et al. Coexistence of rare arteries in the human celiaco-mesenteric system. Acta Med Okayama. 1998; 52(5): 239-244.

12. Nayak $S$, Shetty $S$, Sirasanagandla $S$, et al. Anomalous origin and vulnerable course of left colic artery in relation to the pancreas: a case report. Forensic Med Anat Res. 2013; 01(04): 83-86, doi: 10.4236/fmar.2013.14018.

13. Nayak S, Sr S, George B, et al. Unusual origin of left colic artery from the superior mesenteric artery and its course across the left kidney. Open Access Case Reports. 2014; 3(4).

14. Noussios G, Dimitriou I, Chatzis I, et al. The main anatomic variations of the hepatic artery and their importance in surgical practice: review of the literature. J Clin Med Res. 2017; 9(4): 248-252, doi: 10.14740/jocmr2902w, indexed in Pubmed: 28270883.

15. Olewnik $Ł$, Wysiadecki G, Polguj M, et al. A rare anastomosis between the common hepatic artery and the superior mesenteric artery: a case report. Surg Radiol Anat. 2017; 39(10): 1175-1179, doi: 10.1007/s00276-017-1859-2, indexed in Pubmed: 28432408.

16. Rusu MC, Vlad M, Voinea LM, et al. Detailed anatomy of a left accessory aberrant colic artery. Surg Radiol Anat. 
2008; 30(7): 595-599, doi: 10.1007/s00276-008-0362-1, indexed in Pubmed: 18483690.

17. Shaikh H, Wehrle CJ, Khorasani-Zadeh A. Anatomy, abdomen and pelvis, superior mesenteric artery. StatPearls, Treasure Island 2021.

18. Stimec BV, Terraz S, Fasel JHD. The third time is the charm-anastomosis between the celiac trunk and the left colic artery. Clin Anat. 2011; 24(2): 258-261, doi: 10.1002/ ca.21081, indexed in Pubmed: 21322049.

19. Tandler J. Uber die Varietäten der Arteria coeliaca und deren Entwickelung. Anat Hefte. 1904; 25(2): 473-500, doi: 10.1007/bf02300762.

20. Testut L, Latarjet A. Traité d'anatomie humaine. Doin, Paris 1948. 